\title{
Beta-cell ontogeny: growth and death
}

\author{
J . C orbett ${ }^{1}$, P.Serup ${ }^{2}$, S. B onner-Weir ${ }^{3}$, J.H . N ielsen ${ }^{2}$ \\ ${ }^{1}$ Department of Pathology, Washington University School of Medicine, St. Louis, Missouri, USA \\ ${ }^{2}$ Hagedorn Research Institute, Gentofte, Denmark \\ ${ }^{3}$ Joslin Diabetes Center, Boston, Massachusetts, USA
}

Our understanding of the birth and death of the pancreatic beta cell is of obvious importance to the cure of diabetes mellitus: whether to expand tissue for transplantation, to bioengineer new cells or to induce islet regeneration in vivo. The scientific interest in this question has risen in recent years, and this session focuses on some of the important issues.

Co-Chair Susan Bonner-Weir briefly introduced the topic of beta-cell growth. For many years beta cells were considered to be terminally differentiated and unchanging after birth. However based on experimental animal models the concept that the mass of beta cells is not static but dynamic with the ability to compensate (increase or decrease) in an effort to maintain glucose homeostasis has been proposed [1]. In contrast to the massive destruction of the beta cells resulting in insulin-dependent diabetes mellitus (IDDM), a normal slow turnover of beta cells is achieved by the careful balance of beta-cell birth and beta-cell death by apoptosis. It is important to remember that cell birth has two components: 1 ) differentiation from undifferentiated precursor cells (neogenesis) as in embryonic development or as can be induced in the adult, and 2) replication of the differentiated beta cell. The excitement in this field is that the molecular control of these processes is beginning to unfold; this excitement is reflected in this session.

The presentations and discussion on beta-cell birth and death focused on transcription factors, external

Participants: H. Edlund, Department of Microbiology, University of Umeå, Sweden

R. Scharfmann, INSERM, Hôpital Robert Debré, France

J. Habener, Laboratory of Molecular Endocrinology,

Massachusetts General Hospital, Howard Hughes

Medical Institute, Harvard Medical School, USA

Corresponding author: P.Serup, Hagedorn Research Institute Niels Steensensvej 6, DK-2820 Gentofte, Denmark cues such as mesenchymal interactions, matrix elements, cytokines and growth factors. These topics overflowed to other sessions (German, Hayek, Newgard, Efrat). The underlying questions addressed included:

1. What directs differentiation of beta cells from the undifferentiated precursor cells?

2. What allows the maintenance of a differentiated phenotype for beta cells?

3. Can we ultimately make new beta cells from precursor cells? from pre-existing beta cells or even from unrelated, non-pancreatic cells?

4. Are there reasons that beta cells are more susceptible to injury/death than most other cells?

As a start Dr. Bonner-Weir noted some of the past advances in this field. While a number of exciting papers were written on pancreatic morphogenesis in the 1960s and 1970s, the chapter by Pictet and Rutter in 1972 [2] is generally considered the seminal paper in this field. In the mid 1980s and early 1990s regeneration, growth and expansion of the beta cells in adult rodents in vivo by neogenesis and/or enhanced replication had been reported after partial pancreatectomy, ductal ligation, cellophane wrapping and overexpression of growth factors/cytokines in transgenic mice. In 1988 two groups showed a transient co-localization of glucagon and insulin during embryonic development of rodent pancreas. These papers were the first of many presenting a schema of cell lineage for the endocrine pancreas. Even though there is now agreement that peptide YY is the first islet hormone expressed and that islet cells go through a transitional stage of expressing more than one hormone, the question of lineage and markers of it is still not resolved. In 1991 Dudek and co-authors [3] showed islet neoformation from transplanted adult rat ducts stripped of their mesenchyme and wrapped in fetal 
gut mesenchyme, showing that adult pancreatic ducts could still undergo differentiation into islets.

Over these same years transcription factors have been identified in the pancreas with varying degrees of restrictive expression to specific cell types. The ability to overexpress or "knock out" genes opened new doors of investigation in this area. The 1994 publication from the Edlunds [4] on the mice with null allele of the transcription factor, pdx-1 was a startling and important milestone (see below). That paper has stimulated renewed interest in the field.

\section{Helena Edlund: Islet cell ontogeny}

Helena Edlund presented the results of a series of experiments aimed at determining the role of the homeodomain transcription factors PDX-1 and ISL1 in pancreatic development.

PDX - 1 deficient mice. At present, very little is known about the sources and the nature of the inductive signals that control the development of the pancreas and the generation of the individual cell differentiated types. Great progress, though, has been made in the identification of transcription factors involved in pancreatic development. The study of cell-specific regulation of the insulin and somatostatin genes have led to the cloning of cDNAs encoding mouse [5] and rat [6] homologues of the homeodomain protein PDX-1 (also known as IPF1, IDX-1 STF-1, IUF-1, and GSF). Helena Edlund reported the results of an elegant series of experiments combining gene targeting and in vitro culture of pancreatic explants. PDX-1 expression is initiated in the epithelium of the primitive foregut in a restricted area determined to develop into the pancreas. When this expression is abrogated by gene targeting the mutant pups survive fetal development but die within a few days after birth [4]. Remarkably, the pups lack the pancreas completely. The gastrointestinal tract and all other internal organs were normal in appearance. Other groups, however, have reported reduced numbers of endocrine cells in the duodenum and the antral part of the stomach. Further analysis revealed that the early inductive events leading to the formation of the pancreatic buds and the appearance of the early insulin and glucagon cells occur in PDX-deficient embryos. However, the subsequent morphogenesis of the epithelium and the progression of differentiation are arrested in PDX-1 deficient embryos. It was also shown that the pancreatic epithelium in the PDX-1 mutants is unable to respond to the signal(s) derived from the mesenchyme which normally promotes pancreatic morphogenesis. Taken together, these data provide evidence that PDX-1 acts cell-autonomously and that it is a defect in the pancreatic epithelium that results in the apancreatic phenotype.
ISL 1 deficient mice. Previous analysis, by Edlund, of cis-elements and transacting factors responsible for the cell-specific expression of the insulin gene resulted in the isolation of a cDNA encoding Islet-1 (ISL1), a LIM-homeodomain protein capable of interaction with an insulin gene cis-element. The great similarity of ISL1 with the Caenorhabditis elegans proteins mec-3 and lin-11, which control cell fate decisions, suggests that ISL1 could be involved in the specification of the pancreatic endocrine cell lineage. Dr. Edlund showed that embryonic expression of ISL1 is initiated soon after the endocrine cells have left the cell cycle. ISL1 is also expressed in the mesenchymal cells that surround the dorsal but not the ventral evagination of the gut endoderm that together comprise the pancreatic anlage.

Dr. Edlund reported analyses of acinar and islet differentiation in mice lacking ISL1. In addition to defects outside the pancreas ISL1 mutant embryos do not form the dorsal pancreatic mesenchyme and there is a failure of exocrine cell differentiation in the dorsal but not the ventral pancreas [7]. In addition, there is a complete loss of endocrine cells in both portions. Importantly, exocrine, but not endocrine, cell differentiation in the dorsal pancreas can be rescued by provision of mesenchyme derived from wild-type embryos. The source of mesenchyme is not required to be pancreatic as liver and lung derived mesenchyme also rescues exocrine cell differentiation. These results show first that ISL1 by virtue of its requirement for the formation of dorsal mesenchyme, is necessary for development of the dorsal exocrine pancreas and second that ISL1 function is required in pancreatic endodermal cells for generation of endocrine cells.

During the discussion it was asked whether any transcription factor regulating expression of adhesion molecules has been identified. Dr. German answered that Pax-6 regulates N-CAM and N-cadherin expression.

Jens Høiriis Nielsen: Beta-cell growth factors and their receptors

Jens Høiriis Nielsen gave an overview of beta-cell growth factors and their receptors with special emphasis on growth hormone $(\mathrm{GH})$ and prolactin (PRL).

Role of $\mathrm{GH}$ and $\mathrm{PRL}$ in beta-cell growth: Growth hormone $(\mathrm{GH})$ is considered as a diabetogenic hormone since it induces insulin resistance and hyperglycaemia by counteracting the effect of insulin on glucose metabolism. There is, however, evidence for a direct trophic effect of $\mathrm{GH}$ on the pancreatic beta cells. Culture of rat islets in the presence of GH, PRL or placental lactogen (PL) was found to stimulate both 
DNA synthesis and insulin production. Not only are the circulating levels of PL, PRL and placental GH elevated during pregnancy but also the expression of their receptors $(\mathrm{R})$ in the islets. Thus, both GH-R and PRL-R mRNA levels are increased two to fivefold in the pancreas from pregnant rats. The expression of the receptors is under different hormonal regulation. Whereas the GH-R is upregulated by glucocorticoids and progesterone, the PRL-R is markedly upregulated by PRL and GH [8].

The effect of GH or PRL on adult human islets is much less than on rat islets, probably because of the low level of receptor expression. This observation reflects the species and age variation of the diabetogenic effect of $\mathrm{GH}$. Thus $\mathrm{GH}$ is not diabetogenic in the intact rat, pregnant dogs and puppies but is diabetogenic in the adult dog and man. Molecular dissection of the GH-R has revealed that distinct domains of the cytoplasmic part are required for various signalling pathways. Thus the proximal proline rich domain (box-1) is necessary for binding and activation of the tyrosine kinase JAK-2, which phosphorylates the signal transducers and activators of transcription proteins STAT-1 and 3, both involved in activation of the fos gene and thereby in cell proliferation. The distal part of the cytoplasmic moiety contains three distinct tyrosine residues which upon phosphorylation can bind STAT-5, which is subsequently phosphorylated, probably by JAK-2, and translocated to the nucleus where it binds to specific DNA sequences in the insulin gene.

Possible role of preadipocyte factor-1 in beta cell growth: In contrast to many other tissues the effect of GH on islets does not seem to be mediated by insulin-like growth factor (IGF)-1. This, however, does not exclude that other autocrine growth factors are induced by $\mathrm{GH}$ in the beta cell. In order to identify genes in the beta cell which are upregulated by $\mathrm{GH} /$ PRL a cDNA library constructed from hGH stimulated neonatal rat islets was screened with cDNA probes from unstimulated and hGH stimulated islets. Clones only hybridizing with hGH stimulated probe were isolated and sequenced. One particular clone homologous to mouse preadipocyte factor-1 (Pref-1) and human fetal antigen-1 (FA-1) was further investigated. The protein contains six epidermal growth factor (EGF) like repeats, similar to the D rosophila proteins Notch and delta which are involved in cell fate determination. Both in man and in rat FA-1 is expressed in most epithelial cells early in development but becomes restricted to the beta cell at a later stage. In adult human beta cells FA-1 immunoreactivity is located in the insulin granules. The function of Pref-1/FA-1 is still unknown but it may be speculated that Pref-1 participates in cell-cell interaction during early development, in analogy with the deltaNotch interaction in D rosophila, preventing terminal differentiation and allowing cell proliferation. Whether Pref- 1 has such a role must await further studies, but the correlation between beta cell proliferation and Pref-1 expression, i.e. late fetal and neonatal islets, islets from pregnant rats and islets exposed to GH and PRL in vitro, support this hypothesis. The question if Pref- 1 could be a marker for duct precursor cells was discussed. The need for such markers was stressed. Newgaard raised the question whether dexamethasone induced ductal proliferation was mediated through a GH-receptor dependent mechanism. It was stated that hypopituitarism does not lead to diabetes, arguing against the idea of $\mathrm{GH}$ as a beta-cell growth factor. A comment to this argument was that a high prevalence of diabetes is observed in the Laron syndrome (dwarfism caused by GH-receptor mutations). Dr. Halban asked whether Pref-1 was sialylated but this was not known at present.

\section{Raphael Scharfmann: Similarities between beta and neuronal cells: possible clues to understand beta-cell development}

A discussion of the mechanisms of differentiation of beta cells, and the similarities of beta-cell differentiation with neuronal cells was presented by Raphael Scharfmann. Since neuronal and beta cells share the expression of a large number of proteins, the possibility that similar mechanisms regulate differentiation of both cell types was discussed. Dr. Scharfmann focused on three main questions: 1) do neurotrophins, which are important for neuronal cell maturation, participate in beta-cell differentiation; 2) do the transcriptional mechanisms thought to be specific for neuronal cells, function in beta cells; 3 ) do endocrine pancreatic cells develop by a default mechanism as is thought to occur during neuronal differentiation.

N eurotrophic factors and beta cells: The soluble neurotrophic factors nerve growth factor (NGF) brain derived neurotrophic factors (BDNF) and neurotrophin-3 (NT3) participate in growth and survival of neuronal cells. These neurotrophins bind to the Trk family of cell surface receptors (Trk-A, Trk-B, TrkC). The rat insulinoma INS-1 cells express a functional high affinity NT3 receptor Trk-C, and NT3 stimulates $\mathrm{Ca}^{2+}$ influx in these cells. These findings suggest that NT3 may modulate beta-cell physiology. NGF receptors (Trk-A) are also expressed on ISN-1 cells and on adult rat beta cells. During embryogenesis immature ductular cells that differentiate into beta cells, express NGF receptors [9]. In addition, evidence was presented that fetal rat beta cells express NGF receptors following a 7 day culture. In addition, NGF is also produced by mesenchyme which surrounds islets, thus NGF may act on islets. Inhibition of NGF receptor signalling using the selective inhibitor K252, 
attenuates islet morphogenesis, implicating NGF in islet development.

Transcriptional mechanisms, thought to be neuronal cell specific, are functional in beta cells: NRSF/REST is a negative regulator of neuronal fate that is known to silence specific neuronal genes in non-neuronal cells. However, in both PC12 and INS-1 cells the mRNA expression and functional activity of NRSF/ REST is absent. This is also the case in three additional insulin or glucagon producing cell lines. Genes expressed in both neuronal and beta cells, including the sodium channel type II, dopamine $\beta$ hydroxylase, neurofilament, and glutamate receptors, are known to be repressed by REST in non-neuronal cells. These studies [10] support a common mechanism of transcriptional regulation in beta cells and neuronal cells, and this transcriptional regulation may be of importance for beta cell development.

Do endocrine cells develop by a default mechanism? Dr. Scharfmann presented evidence for a default pathway in pancreatic endocrine development. During initial differentiation (E10-E12), the first immature insulin-expressing cells develop (expressing both insulin and glucagon, but not GLUT2); however, these cells do not associate to form islets. During a second transition (E13-E20), the number of mature insulin containing cells (glucagon negative and GLUT2 positive) increase rapidly and these cells form islets. It is well established that the initial differentiation requires the presence of mesenchyme; however, Scharfmann provided evidence that the second transition of beta cells occurs by mesenchyme-independent mechanisms. E12.5 pancreatic epithelia grown in the presence of its surrounding mesenchyme results in full differentiation of the exocrine component. However, the endocrine cells develop poorly and are immature as judged by co-expression of insulin and glucagon and lack of GLUT2 expression. In contrast, in the absence of mesenchyme, exocrine tissue develops poorly while endocrine cells increase. These endocrine cells do not co-express insulin and glucagon but do express GLUT2 and form islet-like aggregates with glucagon-producing cells in the periphery surrounding a core of insulin-producing cells. These findings prompted Dr. Hayek to suggest that this effect of mesenchyme may explain why his group found less islet development with increasing amounts of mesenchyme transplanted with human fetal isletlike cell clusters (ICCs). Importantly, these findings suggest that mesenchyme may exert a repressive role on the development of pancreatic endocrine tissue. Thus, pancreatic islets may develop by default, i.e. in the absence of a signal endocrine tissue will develop, while in the presence of a signal (mesenchyme) endocrine development is retarded and exocrine tissue develops.
The difficulty in removing all of the mesenchyme was acknowledged, but on the basis of vimentin amounts to only $2 \%$ of the mesenchyme which remained on these buds/explants. However the media did contain serum so there was some growth of the stripped epithelium. Dr. Newgaard asked if a possible effect of mesenchyme on REST expression had been investigated, but this was not yet the case.

\section{Joel Habener: Role of transcription factors in} beta-cell turnover and insulin gene regulation

A transcription factor hypothesis was proposed by Joel Habener as a potential mechanism contributing to the impaired insulin production and reduced betacell mass manifested in non-insulin-dependent diabetes mellitus (NIDDM). The working model implicates two transcription factors, $\mathrm{PDX}-1$ and $\mathrm{C} / \mathrm{EBP} \beta$, having opposing functions in the regulation of insulin gene expression, the differentiation and programmed cell death (apoptosis) of beta cells.

$\mathrm{C} / \mathrm{EBP} \beta$ inhibits insulin gene transcription. $\mathrm{C} / \mathrm{EBP} \beta$, a basic leucine zipper (bZIP) transcription factor, a socalled early response protein induced by cellular stress downregulates insulin gene regulation and may be involved as an upstream effector of the apoptosis pathway. Several sequences in the insulin promoter bind recombinant $\mathrm{c} / \mathrm{EBP} \beta$ when analysed in DNase I footprinting analysis. A functional role for $\mathrm{C} / \mathrm{EBP} \beta$ is indicated by data from transient transfections of $\beta$ TC6 cells in which expression of an insulin reporter construct is inhibited by co-transfected $\mathrm{C} /$ EBP $\beta$. In contrast, when the experiment is performed in non-beta-cells such as BHK and HeLa insulin promoter activity is strongly induced. These apparent paradoxical effects might be explained by the selective presence of PDX-1 in the $\beta$ TC6 cells and suggest that somehow $\mathrm{C} / \mathrm{EBP} \beta$ interferes with the function of PDX on the insulin promoter. The inhibition seen in $\beta$ TC6 cells does not appear to be mediated through direct DNA-binding to the insulin promoter as cotransfection of $\mathrm{CHOP}$, a dominant negative inhibitor of $\mathrm{C} / \mathrm{EBP} \beta$ DNA-binding, failed to abrogate the effect of $C / E B P \beta$. Rather, Habener suggested that interaction of $\mathrm{C} / \mathrm{EBP} \beta$ with $\mathrm{E} 47$, another insulin gene transcription factor, through the leucine zippers present in both factors disrupted the synergy between PDX-1 and the helix-loop-helix IEF-1 of which E47 is a component. Antibodies to $\mathrm{C} / \mathrm{EBP} \beta$ immunoprecipitated E47 from $\beta$ TC6 nuclear extracts showing a direct protein-protein interaction.

PDX - 1 and C/E BP $\beta$ in "glucose toxicity". It is well recognized that chronic hyperglycaemia of NIDDM impairs insulin production and secretion, a phenomenon referred to as "glucose toxicity". This impairment 
may be a consequence of chronic hyperglycaemia on the expression of PDX-1 and C/EBP $\beta$. A number of models, in vitro and in vivo, were analysed for expression of PDX-1 and C/EBP $\beta$. Insulinoma cells grown long term (HIT, several weeks) at elevated glucose levels $(25 \mathrm{mmol} / \mathrm{l})$ or short term (INS-1, $72 \mathrm{~h}$ ) exhibit progressive induction of $\mathrm{C} / \mathrm{EBP} \beta$ and inhibition of PDX expression. Similarly in vivo models of chronic hyperglycaemia, the 12-week-old Zucker fatty diabetic (ZDF) rat and the hyperglycaemic rat 4 weeks after partial pancreatectomy, had decreased PDX-1 and increased $\mathrm{C} / \mathrm{EBP} \beta$ expression. Habener proposed that chronic hyperglycaemia acts as a metabolic stress to beta cells resulting in the induction of the expression of $\mathrm{C} / \mathrm{EBP} \beta$ and the consequent impairment of insulin gene expression. The mechanisms through which chronic hyperglycaemia inhibit PDX1 expression remain unknown. An additional component of these studies is the implication that chronic hyperglycaemia may impair neogenesis and accelerate apoptosis of beta cells via effects on the expression of PDX-1 and C/EBP $\beta$ contributing to a reduction in beta-cell mass, a characteristic occurrence in severe NIDDM.

During the discussion it was mentioned that a $\mathrm{C} /$ EBP family member was recently found to be activating the transcription of the C. elegans apoptosis promoting factors ced 3 and ced 4 . The role of survival factors such as bcl-2 family or NGF was discussed. Additionally, the direct effects of glucose concentrations on binding of PDX-1 to insulin promoter were discussed.

\section{John Corbett: Cytokine mediated beta-cell damage}

In the end of this session, findings which support a role for cytokine-induced beta-cell damage and new studies on the mechanisms of cytokine mediated beta-cell destruction were presented by John Corbett. A large body of evidence supports the direct role for cytokine-induced damage as one mechanism leading to beta-cell destruction in autoimmune diabetes. Recent studies by Unger and co-workers suggest that cytokine-induced beta-cell damage may also participate in beta-cell damage or "burn-out" in obesity models of NIDDM (see sections on fatty acids and beta-cell function).

D o cytokines mediate beta-cell damage? MandrupPoulsen and Nerup first showed in 1985 that treatment of rat islets with interleukin-1 (IL-1) results in an inhibition of insulin secretion that is followed by islet damage after prolonged exposures (4-6 days); reviewed previously [11]. These initial studies provided the first evidence that cytokines could directly inhibit the function of beta cells. Recent studies indicate that the mechanism by which IL- 1 mediates beta-cell damage is a result of inducible nitric oxide synthase (iNOS) expression and the increased production of the free radical nitric oxide.

Islet cellular source and cytokine requirements for iNOS expression. An important question concerning the specificity of cytokine-induced islet damage is the islet cellular source of iNOS. We (Corbett et al.) have shown by immunohistochemical co-localization that treatment of rat islets with IL-1 results in the expression of iNOS by insulin containing cells, and that non-insulin containing cells do not express this enzyme [12]. These findings support the hypothesis that IL-1-induced islet damage is mediated by selective expression of iNOS by beta cells. The cytokine requirements for beta-cell expression of iNOS have been examined. IL-1 is sufficient to stimulate iNOS expression by rat beta cells, while a combination of IL- 1 and interferon- $\gamma$ (IFN- $\gamma$ ) are required to stimulate iNOS expression by NOD mouse and human beta cells.

Mechanisms of cytokine-induced beta-cell damage: Cytokine-induced beta-cell damage is mediated, in part, by nitric oxide-induced inhibition of mitochondrial glucose oxidation (specifically the Krebs cycle enzyme aconitase, and the electron transport chain complex 1 and 2), and DNA damage. DNA damage may be mediated by both nitric oxide-dependent and -independent mechanisms, while IL-1-induced inhibition of mitochondrial function appears to be associated with nitric oxide-induced iron-sulphur centre destruction of the indicated enzyme targets. The free radical species that mediates IL-1-induced betacell damage has been controversial. Nitric oxide, oxygen radicals, and/or peroxynitrite (produced following the interactions of nitric oxide and superoxide) have been implicated in both IL-1 and IL-1 + IFN- $\gamma$ induced beta-cell damage in rat and human islets. Current studies indicate that human and rat islets are equally sensitive to peroxynitrite while rat islets are more sensitive to nitric oxide than human islets. The nitrogen oxide species that mediates beta-cell damage and the source and type of oxygen radicals that are produced by beta cells in response to IL-1 have yet to be identified.

Role of cytokine-induced beta-cell damage in the development of autoimmune diabetes: Resident islet macrophages are believed to be an islet cellular source of IL-1. Treatment of rat islets with TNF + LPS, a condition known to activate macrophages, stimulates iNOS expression, nitrite formation, and inhibits glucose-induced insulin secretion. The IL-1 receptor antagonist protein (IRAP) completely prevents TNF + LPS-induced inhibition of insulin secretion and attenuates iNOS expression and nitric oxide production by rat islets. Immunohistochemical 
analysis reveals that TNF + LPS stimulate the expression of iNOS by insulin positive cells and a second population of cells believed to be resident islet macrophages. IRAP completely prevents TNF + LPS-induced iNOS expression by beta cells but does not inhibit the expression by resident islet macrophages [12]. These new findings indicate that the activation of a limited number of resident islet macrophages (approximately 10-15/islet) results in the release of IL-1, and that the levels of IL-1 produced in the microenvironment of the islet are sufficient to stimulate iNOS expression by beta cells. Thus, resident macrophage activation may be one potential initiation event that stimulates the initial destruction of beta cells.

\section{Future directions and recommendations}

The Chairs and rapporteurs of this session have selected the following list of recommendations as topics for future lines of investigation:

- Identification of the sources and nature of the inductive signals that controls pancreas morphogenesis and cytodifferentiation.

- Identification and function of transcription factors involved in beta-cell development.

- Identification of an islet precursor/stem cell.

- Identification of genes expressed during beta-cell growth and regeneration.

- Study the mechanisms involved in beta-cell growth during pregnancy.

- Search for beta-cell regeneration in pathological states in man, e.g. chronic pancreatitis, obesity, IDDM and NIDDM.

- Establishment of an inventory of syndromes affecting the endocrine pancreas in order to identify the genes involved.

- A search for mutations in genes involved in betacell differentiation and growth and their association with IDDM and NIDDM.

- Resolving the mechanism of "glucose toxicity" and means to prevent it.

- Clarification of the molecular mechanisms involved in cytokine-induced beta-cell killing and means of prevention.
- A search for mutations in genes involved in betacell destruction and defence and their association with IDDM.

\section{References}

1. Finegood D, Scaglia L, Bonner-Weir S (1995) Dynamics of beta-cell mass in the growing rat pancreas. Estimation with a simple mathematical model. Diabetes 44: 249-256

2. Pictet R, Rutter WJ (1972) Development of the embryonic endocrine pancreas. In: Steiner DF, Freinkel N (eds) Handbook of physiology, section 7: Endocrinology, Volume 1. American Physiological Society Washington DC: pp 25-66

3. Dudek RW, Lawrence J, Hill RS, Johnson RC (1991) Induction of islet cytodifferentiation by fetal mesenchyme in adult pancreatic ductal epithelium. Diabetes 40: 1041-1048

4. Jonsson J, Carlsson L, Edlund T, Edlund H (1994) Insulin promoter factor-1 is required for pancreas development in mice. Nature 371: 606-609

5. Ohlsson H, Karlsson K, Edlund T (1993) IPF1, a homeodomain-containing transactivator of the insulin gene. EMBO J 12: 4251-4259

6. Miller CP, McGehee RE, Habener JF (1994) IDX-1: a new homeodomain transcription factor expressed in rat pancreatic islets and duodenum that transactivates the somatostatin gene. EMBO J 13: 1145-1156

7. Ahlgren U, Pfaff SL, Jessell TM, Edlund T, Edlund H (1997) Independent requirement for ISL1 in formation of pancreatic mesenchyme and islet cells. Nature 385: 257-260

8. Møldrup A, Petersen E, Nielsen J (1993) Effects of sex and pregnancy hormones on growth hormone and prolactin receptor gene expression in insulin-producing cells. Endocrinology 133: 1165-1172

9. Kanaka-Gantenbein C, Tazi A, Chernichow P, Scharfmann $R$ (1995) In vivo presence of the high affinity nerve growth factor receptor Trk-A in the rat pancreas: differential localization during pancreatic development. Endocrinology 136: 761-769

10. Atouf F, Chernichow P, Scharfmann R (1997) Expression of neuronal traits in pancreatic beta cells. Implication of neuron-restrictive silencing factor/repressor element silencing transcription factor, a neuron-restrictive silencer. $\mathbf{J}$ Biol Chem 272: 1929-1934

11. Mandrup-Poulsen T (1996) The role of interleukin-1 in the pathogenesis of IDDM. Diabetologia 39: 1005-1029

12. Corbett J, McDaniel M (1995) Intraislet release of interleukin 1 inhibits $\beta$-cell function by inducing $\beta$-cell expression of inducible nitric oxide synthase. J Exp Med 181: 559-568 